\title{
A prognostic model based on cell-cycle control predicts outcome of breast cancer patients
}

Heli Repo ${ }^{1,2}$, Eliisa Löyttyniemi ${ }^{3}$, Samu Kurki ${ }^{4}$, Lila Kallio ${ }^{4}$, Teijo Kuopio ${ }^{2}$, Kati Talvinen ${ }^{1}$ and Pauliina Kronqvist ${ }^{1,5^{*}}$ (D)

\begin{abstract}
Background: A prognostic model combining biomarkers of metaphase-anaphase transition of the cell cycle was developed for invasive breast cancer. The prognostic value and clinical applicability of the model was evaluated in comparison with the routine prognosticators of invasive breast carcinoma.

Methods: The study comprised 1135 breast cancer patients with complete clinical data and up to 22-year followup. Regulators of metaphase-anaphase transition were detected immunohistochemically and the biomarkers with the strongest prognostic impacts were combined into a prognostic model. The prognostic value of the model was tested and evaluated in separate patient materials originating from two Finnish breast cancer centers.

Results: The designed model comprising immunoexpressions of Securin, Separase and Cdk1 identified 8.4-fold increased risk of breast cancer mortality $(p<0.0001)$. A survival difference exceeding 15 years was observed between the majority (> 75\%) of patients resulting with favorable as opposed to unfavorable outcome of the model. Along with nodal status, the model showed independent prognostic impact for all breast carcinomas and for subgroups of luminal, $\mathrm{N}+$ and $\mathrm{N}$ - disease.

Conclusions: The impact of the proposed prognostic model in predicting breast cancer survival was comparable to nodal status. However, the model provided additional information in $\mathrm{N}$ - breast carcinoma in identifying patients with aggressive course of disease, potentially in need of adjuvant treatments. Concerning $\mathrm{N}+$, in turn, the model could provide evidence for withholding chemotherapy from patients with favorable outcome.
\end{abstract}

Keywords: Breast cancer, Prognosis, Proliferation, Cell cycle, Securin, Separase

\section{Background}

Cell proliferation, hormone-regulation and HER2 amplification are considered the main biological processes driving breast cancer progression. Although proliferation has been shown a valid prognosticator in all subtypes, particularly triple-negative breast carcinoma (TNBC) has been characterized by high expression of proliferationrelated genes $[1,2]$. The prognostic value of proliferation

\footnotetext{
* Correspondence: paukro@utu.fi

${ }^{1}$ Institute of Biomedicine, University of Turku, Turku, Finland

${ }^{5}$ Department of Pathology, University of Turku, Kiinamyllynkatu 10/MedD5A, 20500 Turku, Finland

Full list of author information is available at the end of the article
}

is acknowledged in the clinical pathology practice as part of the traditional histological grading as well as in intrinsic classification and in modern personalized signatures retrieved from microarray-based expression-profiling [3-5]. However, the impact of deregulated proliferation is still not accurately reflected in the routine clinical parameters and pathological markers applied to treatment decisions of breast cancer patients.

Genetic integrity of the dividing cell is ensured by complex and intricately monitored cellular events at the metaphase-anaphase transition of the cell cycle [6]. Dysfunction of these regulators can lead into missed sister

(c) The Author(s). 2020 Open Access This article is licensed under a Creative Commons Attribution 4.0 International License, which permits use, sharing, adaptation, distribution and reproduction in any medium or format, as long as you give appropriate credit to the original author(s) and the source, provide a link to the Creative Commons licence, and indicate if changes were made. The images or other third party material in this article are included in the article's Creative Commons licence, unless indicated otherwise in a credit line to the material. If material is not included in the article's Creative Commons licence and your intended use is not permitted by statutory regulation or exceeds the permitted use, you will need to obtain permission directly from the copyright holder. To view a copy of this licence, visit http://creativecommons.org/licenses/by/4.0/ The Creative Commons Public Domain Dedication waiver (http://creativecommons.org/publicdomain/zero/1.0/) applies to the data made available in this article, unless otherwise stated in a credit line to the data. 
chromatid separation, chromosomal instability and aneuploidy. Premature sister chromatid separation is prevented by the highly controlled sequential activation and inactivation of a cascade of regulatory proteins, particularly Cdc20 (cell division cycle 20), Cohesin, Separase (Extra Spindle Pole Bodies Like protein 1, ESPL1), Securin (Pituitary tumor-transforming gene 1, Pttg1), Pttg1IP (Pituitary tumor-transforming gene 1 interacting protein, also Pituitary tumor-transforming gene 1 binding factor, PBF), Cdk1 (Cyclin-Dependent Kinase protein 1) and CyclinB1 (G2/mitotic-specific cyclin-B1). In more detail, correct segregation of the chromosomes is triggered at the Spindle Assembly Checkpoint (SAC) controlled by $\mathrm{Cdc} 20$ activating Anaphase-Promoting Complex / Cyclosome (APC/C) to create the APC/ $\mathrm{C}^{\mathrm{Cdc20}}$ complex. Throughout metaphase, contact between the chromatids is maintained by rings of Cohesin. At the initiation of anaphase, Cohesin is removed triggered by Separase and activated by degradation of Securin and/or the Cdk1/CyclinB1 complex, leading to separation of the sister chromatids $[7,8]$.

In our previous research, PTTG1, the gene of human Securin, was detected with the most significant expression difference between human breast cancer and normal breast glandular tissue on basis of a cDNA microarray analysis involving 4000 cancer related genes [9]. In addition to Securin, also several other regulators of metaphase-anaphase transition have been shown with independent prognostic impacts in breast cancer [1018]. In the present study, we introduce on basis of a total of 1135 patients with up to 22-year follow-up, a clinically applicable combination of biomarkers of metaphaseanaphase transition leading to optimal detection of aggressive course of disease and cancer mortality in invasive breast cancer.

\section{Methods \\ Patient materials}

The study comprises patients diagnosed and treated with unilateral invasive breast carcinoma in two different institutions (Table 1). The first cohort (I) $(n=781)$ originated from the Central Hospital of Central Finland, Jyväskylä, Finland, from years 1987-1997 resulting in maximum follow-up time of 22.7 years. The second cohort (II) $(n=354)$ was collected from Turku University Hospital and Auria biobank, Turku, Finland. This cohort was classified into intrinsic subgroups comprising 208 patients with luminal and 148 patients with triplenegative breast carcinomas diagnosed and treated during 2005-2015 resulting in maximum follow-up times of 14 and 17.8 years, respectively.

For all patients, the biomarkers of the metaphaseanaphase transition were immunohistochemically (IHC) detected, and the routine clinico-pathological prognostic features of breast cancer were collected. For both cohorts of breast cancer patients, a prognostic model was assembled based on the optimal combination of biomarkers of the metaphase-anaphase transition. The prognostic value of the models was evaluated in comparison with the clinically applied routine prognosticators of breast cancer.

Table 1 Summary of patient cohorts with clinico-patohologic characteristics

\begin{tabular}{|c|c|c|c|}
\hline & \multirow{2}{*}{$\begin{array}{l}\text { Cohort I } \\
\text { All subtypes } \\
(n=781)\end{array}$} & \multicolumn{2}{|l|}{ Cohort II } \\
\hline & & $\begin{array}{l}\text { Luminal } \\
(n=208)\end{array}$ & $\begin{array}{l}\text { TNBC } \\
(n=146)\end{array}$ \\
\hline Mean age at diagnosis (range) (years) & $61(28-95)$ & $62(42-76)$ & $60(39-78)$ \\
\hline Axillary lymph node positive (\%) & 44.8 & 22.8 & 32.1 \\
\hline Mean tumor size (range) (cm) & $2.3(0.2-16.0)$ & $1.9(0.4-7.0)$ & $2.5(0.2-18.0)$ \\
\hline \multicolumn{4}{|l|}{ Histological type (\%) } \\
\hline Infiltrating ductal NOS & 75.4 & 72.3 & 100 \\
\hline Special type & 24.6 & 27.2 & 0 \\
\hline \multicolumn{4}{|l|}{ Intrinsic subtype (\%) } \\
\hline Luminal & 67.6 & 100 & - \\
\hline Her2-amplified & 18.6 & - & - \\
\hline Triple-negative & 13.8 & - & 100 \\
\hline \multicolumn{4}{|l|}{ Histological grade (\%) } \\
\hline Low (1-2) & 79.6 & 81.6 & 0 \\
\hline High (3) & 20.4 & 18.4 & 100 \\
\hline Median follow-up time (max) (years) & $12.4(22.7)$ & $11.8(14.0)$ & $6.9(17.8)$ \\
\hline Dead of breast cancer (\%) & 30.7 & 10.7 & 22.6 \\
\hline
\end{tabular}


All patients were treated with surgical resection or mastectomy with axillary evacuation, radiation and/or adjuvant treatment with anti-estrogenic or cytostatic drugs depending on the patients' age, hormone receptor and lymph node $(\mathrm{N})$ status according to the international guidelines for breast cancer treatment at the time of diagnosis [19]. No pre-operative adjuvant treatments were administered. Complete clinical data was collected from pathology reports and patient files and registered applying the criteria presented by WHO [20] and St. Gallen International Expert Consensus [21]. Intrinsic subtypes were approximated by immunohistochemistry according to international guidelines [22]. Causes of death were obtained from autopsy reports, death certificates and from the national cancer registry (Statistics Finland, Helsinki, Finland).

\section{Tissue materials}

Tissue materials were prepared according to standard histology practice, i.e. fixed in buffered formalin ( $\mathrm{pH}$ 7.0) and embedded into paraffin blocks. Tissue micro arrays (TMAs) were prepared using the representative tumor area of each patient. The TMAs included two tissue cores with diameters $0.6 \mathrm{~mm}$ (cohort I) or $1 \mathrm{~mm}$ (cohort II) from each tumor.

\section{IHC methods}

Immunohistochemistry was performed on sections of TMAs cut at $3 \mu \mathrm{m}$. Immunohistochemical stainings for Securin, Separase, Cdc20, Pttg1IP, SA2 subunit of Cohesin and CyclinB1 were performed as previously described ([9, 13, 14, 16, 17], Additional file 1). IHC for detecting Cdk1 was performed on an automated immunostaining platform Discovery XT (Roche Diagnostics/ Ventana Medical Systems, Tucson, AZ). Deparaffinization, epitope retrieval, and antibody incubation were performed before detection with OmniMap DAB Detection Kit (Roche/Ventana). IHC for Ki-67, estrogen (ER) and progesterone (PR) receptors and HER2, and HER2amplification with in situ hybridization (ISH) followed standard protocols.

\section{Interpretation of IHC}

Immunoexpressions for Securin, Separase, Cdc20, Pttg1IP, SA2, Cdk1 and CyclinB1 were observed as combinations of nuclear and cytoplasmic staining and registered as average fractions (\%) of positively staining cancer cells $[13,14,16,23]$. In each case, the number of immunopositive cells was calculated in sets of one hundred cancer cells (minimum 100 and maximum $3 \times 100$ cancer cells evaluated) and registered as an average fraction (\%) of immunopositivity for each patient. Interpretations for IHC of Ki-67, ER, PR, and IHC and ISH for HER2 followed previous literature and generally accepted international guidelines [22, 24]. All IHC interpretations were performed by experienced histopathologists (HR, PK).

\section{Statistical analysis}

The cutpoints for immunoexpressions of the studied biomarkers were set based on previous literature, histopathological observations and statistical analyses involving the mean, median and univariate prognostic values of each parameter $[9,13,14,16,17]$. In prognostic analyses, Kaplan-Meier estimates were performed to demonstrate the cumulative percentages of breast cancer specific mortality. Cox's proportional hazard models were used to test associations between disease outcome, biomarker expressions and clinical prognostic features, i.e. tumor size, axillary lymph node status, histological and intrinsic classifications and histological grade. The risk of breast cancer death associated with the studied proteins and the routine prognosticators was quantitated as hazard ratio (HR) with $95 \%$ confidence interval (CI). $P$-values $<0.05$ were considered statistically significant. The computations were performed with SAS for Windows, Version 9.3 (I-III) and 9.4 (IV) (SAS Institute, Cary, NC, USA). Kaplan-Meier survival plots were generated using R 2.15.0.

\section{Results}

In IHC of the invasive breast carcinomas, Securin was detected as predominantly nuclear but occasionally, showed both nuclear and/or cytoplasmic

Table 2 Fraction (\%) of carcinomas immunopositive for the studied proteins in the whole material and in subgroups

\begin{tabular}{lllllllllll}
\hline & $\mathrm{n}$ & $\mathrm{All}$ & $\mathrm{N}-$ & $\mathrm{N}+$ & $\mathrm{T} 1$ & $\mathrm{~T} 2-3$ & low grade & high grade & alive & DOD \\
\hline Securin & 604 & 34 & 28 & 52 & 19 & 36 & 28 & 52 & 42 \\
Separase & 401 & 31 & 18 & 32 & 15 & 19 & 17 & 57 & 19 & 18 \\
Cdc20 & 387 & 5 & 3 & 7 & 3 & 6 & 4 & 11 & 4 \\
Pttg1IP & 420 & 56 & 59 & 53 & 65 & 51 & 66 & 28 & 60 \\
SA2 & 470 & 28 & 32 & 22 & 29 & 26 & 28 & 26 & 51 \\
Cdk1 & 384 & 23 & 9 & 24 & 8 & 24 & 12 & 26 & 6 \\
CyclinB1 & 455 & 57 & 53 & 61 & 49 & 60 & 55 & 66 & 6 \\
\hline
\end{tabular}

$N$ - node-negative, $N+$ node-positive, $T 1$ tumor size $<2 \mathrm{~cm}, T 2-3$ tumor size $\geq 2 \mathrm{~cm}$, low grade = grades $1-2$, high grade grade $3, D O D$ dead of disease 

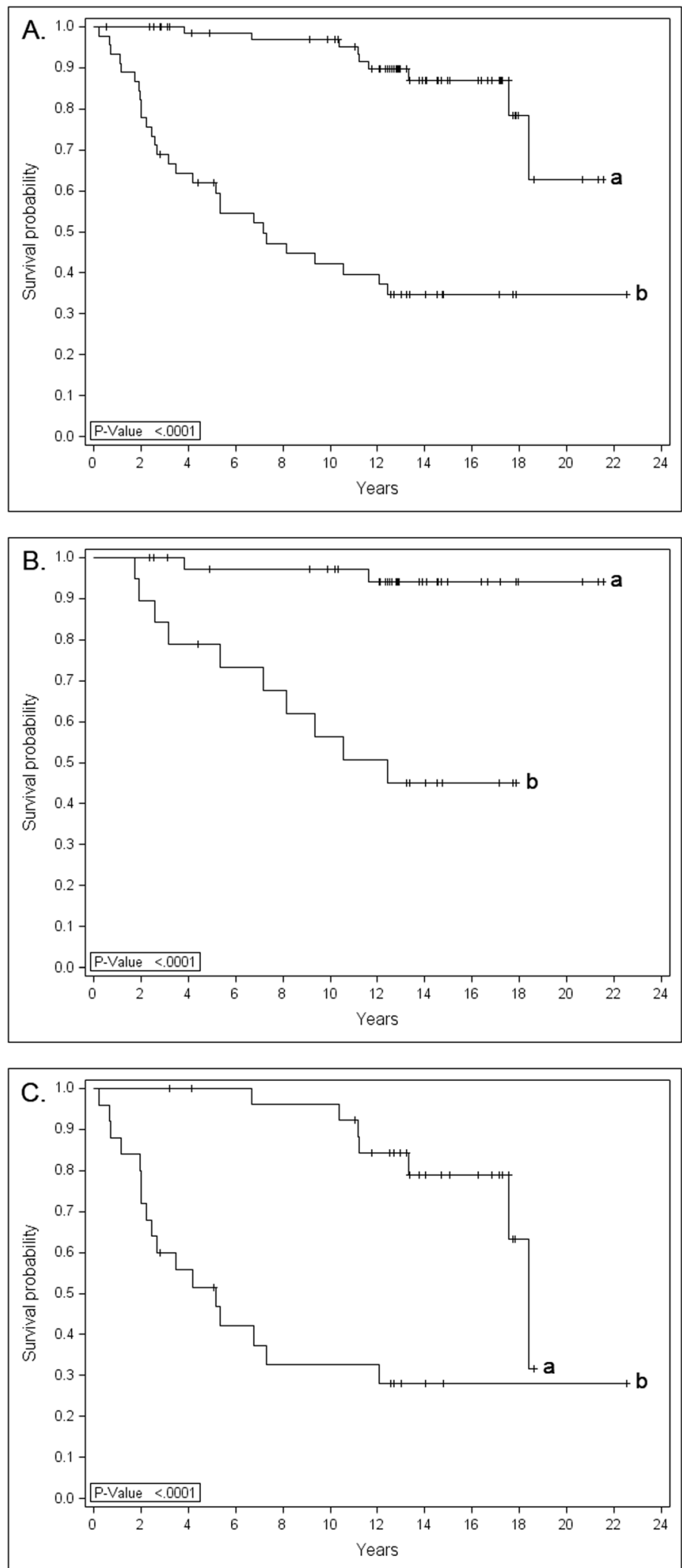

Fig. 1 Kaplan-Meier curves show the survival difference between favorable (curve a: low immunoexpressions for Securin, Separase and Cdk1) vs unfavorable (curve b: high expressions of Securin, Separase and Cdk1) outcome of the prognostic model for all breast carcinomas (a) and for subgroups with $\mathrm{N}+(\mathbf{b})$ and $\mathrm{N}-(\mathbf{c})$ disease (cohort I, $n=781$ ) 
Table 3 Multivariate analyses involving the prognostic model ${ }^{\mathrm{a}}$ with nodal status, tumor size, intrinsic classification and histological grade

\begin{tabular}{|c|c|c|c|}
\hline & $H R^{b}$ & $p$ & $\mathrm{Cl}$ \\
\hline \multicolumn{4}{|l|}{ All patients $(n=781)$} \\
\hline Model & 8.4 & $<0.0001$ & $3.4-20.7$ \\
\hline Nodal status & 4.3 & $<0.0001$ & $2.6-7.0$ \\
\hline Tumor size & & ns. & \\
\hline Tumor grade & & ns. & \\
\hline Intrinsic classification & & ns. & \\
\hline \multicolumn{4}{|l|}{$\mathbf{N}+$ patients $(n=350)$} \\
\hline Model & 6.5 & 0.0003 & $2.3-17.9$ \\
\hline Tumor size & & ns. & \\
\hline Tumor grade & & ns. & \\
\hline Intrinsic classification & & ns. & \\
\hline \multicolumn{4}{|l|}{ N- patients $(n=431)$} \\
\hline Model & 19.5 & 0.006 & $2.3-163.8$ \\
\hline Tumor size & & ns. & \\
\hline Tumor grade & & ns. & \\
\hline Intrinsic classification & & ns. & \\
\hline
\end{tabular}

( $\geq 1 \%$ of cancer cells) and Cdk1 ( $\geq 10 \%$ of cancer cells)

${ }^{a}$ High expression for Securin $(\geq 10 \%$ of cancer cells), Separase

${ }^{\mathrm{b}}$ The hazard ratio of breast cancer death

ns not statistically significant

immunoreaction. Separase showed two distinct and apparently mutually excluding expression patterns observed in the nucleus or in the cytoplasm of cancer cells. Nuclear immunoexpression was observed for Cdc20, SA2, Cdk1 and CyclinB1 whereas Pttg1IP showed cytoplasmic expression only. Table 2 summarizes the fractions of immunopositive breast carcinomas among all breast cancer subtypes (cohort I) and in subgroups divided according to tumor size, nodal status, histological grade and survival.

The prognostic impacts of the studied regulators of metaphase-anaphase transition were first analyzed for all breast cancer subtypes (cohort I, $n=781$ ) with Cox's proportional hazard model. Among all studied biomarkers, statistically significant prognostic value in univariate analyses was observed for Securin (HR 2.1, $p<$ 0.0001, CI 1.6-2.8), nuclear Separase (HR 2.0, $p<$ 0.0004, CI 1.4-3.0), Cdk1 (HR 2.5, $p<0.0001$, CI 1.73.6) and CyclinB1 (HR 2.0, $p=0.04$, CI 1.0-2.0).

In the next phase, these biomarkers were further tested in combinations in order to assemble a prognostic model producing the most significant prognostic impact among all breast cancer subtypes. The optimal model for detecting favorable outcome of disease was determined as the combination of low expressions for Securin $(<10 \%$ of cancer cells), Separase $(<1 \%$ of cancer cells) and Cdk1 $(<10 \%$ of cancer cells). This model was a significant indicator of survival of disease while the opposite detected patients in risk of breast cancer death (HR 8.4, $p<0.0001)$. The Kaplan-Meier curves demonstrate the survival difference among all patients and in subgroups with $\mathrm{N}+$ and $\mathrm{N}$ - disease (Fig. 1). Concluding from the survival analyses, favorable outcome of the model indicated that the majority $(>75 \%)$ of patients were alive 18.4 years after primary diagnosis while unfavorable outcome of the model suggested that one quarter $(25 \%)$ of the patients were already dead of breast cancer after 2.5 years of diagnosis. Among the subgroup of $\mathrm{N}$ - patients, no cancer-related deaths were observed among patients exhibiting favorable outcome of the model. Instead, the unfavorable outcome suggested cancer mortality for every fourth patient within 5.3 years from diagnosis. Correspondingly, the majority of $\mathrm{N}+$ patients with favorable and unfavorable outcome of the model were alive after 17.6 and 2.0 years from the primary diagnosis, respectively.

Finally, in multivariable analyses of all breast cancer subtypes (Table 3 ), the designed model was compared with the established clinical prognosticators of breast cancer, i.e. tumor size, nodal status, intrinsic classification and histological grade for superior prognostic power in predicting the risk of breast cancer mortality. In the whole material, significant prognostic impact was observed for axillary lymph node status along with the designed model. Independent prognostic value was detected for the model also among $\mathrm{N}+$ and $\mathrm{N}$ - patients. Tumor size (diameter $<2 \mathrm{~cm}$ vs $\geq 2 \mathrm{~cm}$ ), intrinsic classification or histological grade (1-2 vs 3 ) were not detected with independent prognostic value in any of the performed analyses.

As the cohort I contained a relatively old patient material, a more recent patient material (cohort II) was collected to evaluate the prognostic impact of the regulators of metaphase-anaphase transition in luminal $(n=208)$ and triple-negative breast carcinomas $(n=146)$. As the result of luminal breast carcinomas, Securin (HR 1.1, $p=0.02$, CI 1.0-1.2) and nuclear Separase (HR 5.7, $p=0.002$, CI 1.9-17.2) - but not Cdk1 remained the most powerful predictors of cancer mortality, along with nodal status (HR 4.9, $p=0.003, \mathrm{CI} 1.7-13.7$ ). Also as combined into a model, Securin and Separase showed significant prognostic impact $(p=0.0006)$. Similar trends for the prognostic model were observed also in separate analyses of $\mathrm{N}+(n=42)$ and $\mathrm{N}-(n=166)$ patients although the associations were not statistically significant in the small patient groups.

In TNBC $(n=146)$, no statistically significant prognostic value could be detected for any of the immunohistochemically studied biomarkers or clinicopathological features. 

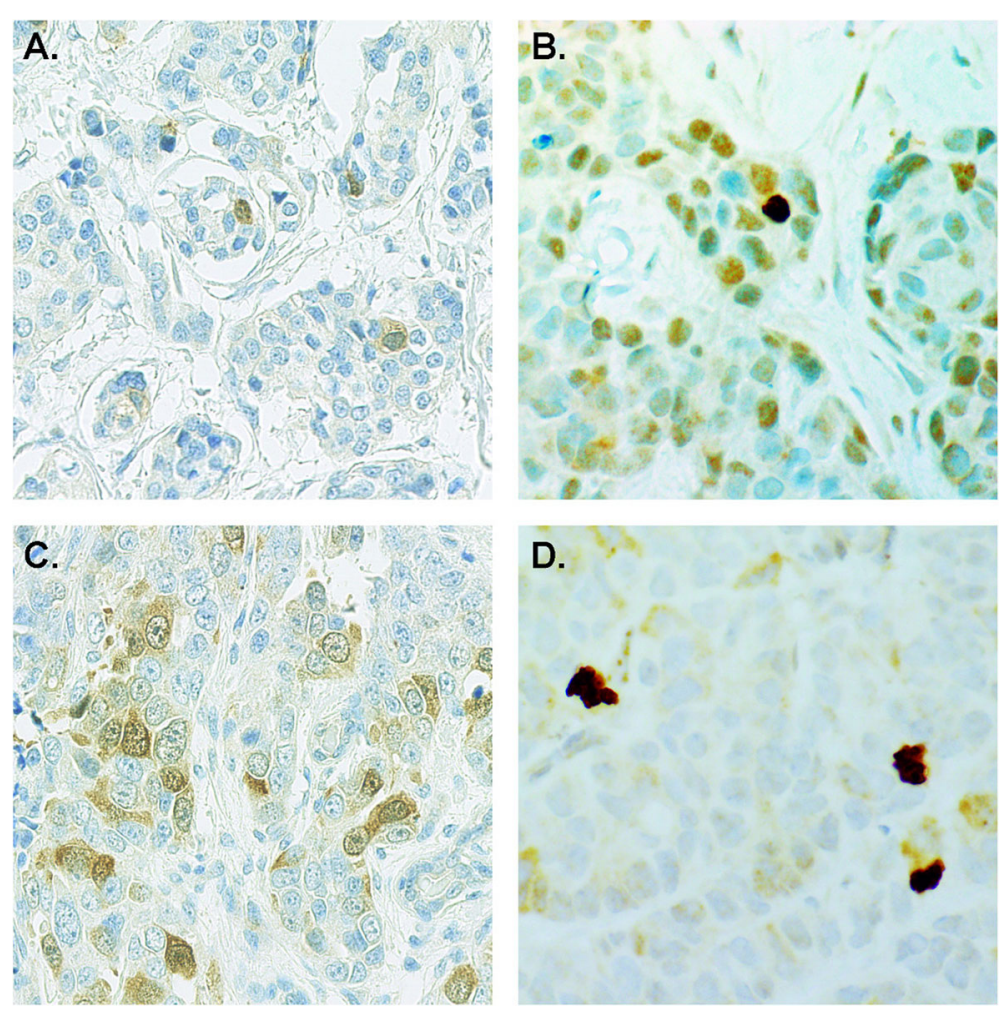

Fig. 2 The combination of immunoexpressions for Securin and Separase indicate favorable (Securin a and Separase b) vs unfavorable (Securin c and Separase d) outcome of breast cancer

\section{Discussion}

In the present study, prognostic models involving regulators of the metaphase-anaphase transition of the cell cycle were introduced for predicting survival of breast cancer patients. The model was assembled based on data from a total of 1135 breast cancer patients with complete clinical information and up to 22-year followup.

The results show that combining high immunoexpressions for Securin, Separase and Cdk1 comprises a promising prognostic model indicating 8.4-fold increased risk of breast cancer death $(p<0.0001)$. In luminal breast carcinomas, the combination of Securin and Separase resulted in independent prognostic impact. In all analyses, the prognostic value of the combination of Securin and Separase with or without Cdk1 outperformed the impact of tumor size and histological grade whereas axillary lymph node status remained a strong and independent prognosticator in all analyses. At highest, the introduced prognostic model predicted 19.5-fold increased risk of breast cancer death among $\mathrm{N}-(p=0.006)$ and 6.5-fold increased risk of mortality among $\mathrm{N}+$ breast carcinomas $(p=0.0003)$. This suggests that the model may provide additional prognostic information to nodal status in treatment decisions of breast cancer patients. Among Ndisease, the model may provide information to identify patients with aggressive course of disease, potentially in need of adjuvant treatments. In $\mathrm{N}_{+}$, the model could provide evidence for withholding chemotherapy from patients with favorable outcome.

TNBC is an aggressive subtype of breast cancer resulting in high mortality. It is also a therapeutically challenging subgroup as the lack of estrogen and progesterone receptors limits the treatment options available. The ongoing intense research has not yet revealed promising novel prognostic biomarkers or treatment targets for TNBC [2]. The present study did not reveal a prognostic impact for the routine clinicopathological parameters or for the studied biomarkers in TNBC. This may be due to the small cohort size as well as the heterogeneous nature of TNBC comprising several molecular subtypes [2].

The value of the introduced model lies in the pivotal role of the studied biomarkers in cancer progression. Loss of control of the cell cycle is a hallmark of malignancy resulting in aneuploidy and genomic instability [25]. Cell cycle checkpoints, including the mitotic checkpoint SAC, are the major cell cycle control mechanism and specifically deregulated in cancer cells. The presently studied regulators of metaphase-anaphase transition are involved in ensuring the fidelity of chromosome segregation. In previous literature, the studied 
biomarkers have been shown with prognostic impact in breast carcinoma as well as in other malignancies [14, 16, 17, 26-33]. In addition, numerous strategies have been proposed for the design of cell cycle-selective therapies in cancer, including targeting the metaphaseanaphase transition [8]. In all, the introduced model appears to identify biological drivers that could add to the conventional histopathological evaluation on the proliferative behavior of the tumor.

The reliability of the introduced prognostic model is increased by repeated statistical analysis of the studied biomarkers. The first approach applied for all breast cancer subtypes (cohort I) was based on testing combinations of biomarkers for their independent prognostic impact in comparison to each other, the clinical prognostic features and disease survival. In the second approach applied for analysis luminal and triple-negative breast carcinomas (cohort II), the optimal combination of biomarkers was extracted together with the clinical prognostic markers in sequential multivariate analyses of breast cancer-specific survival. Each approach was independently applied on separate patient materials originating from two Finnish breast cancer centers. As a conclusion, irrespective of the patient material or statistical procedure, the combination of Securin and Separase showed the most significant prognostic impact among all breast carcinomas and among luminal breast cancer.

From the practical point of view, immunohistochemical detection of Securin and Separase comprises a biology driven, cost-effective and reliable prognostic method (Fig. 2). By comparison, Ki-67 - the established proliferation marker of cancer diagnostics - has been criticized for high variability across individual pathologist and institutions, as well as for poor prognostic value [34, 35]. In the literature, numerous prognostic models have been introduced for breast cancer but only a few of them have shown impact exceeding that of the routine clinical prognosticators [36-38].

\section{Conclusions}

In our results from a total of 1135 breast cancer patients with complete clinical information and up to 22-year follow-up, high immunoexpression for the combination of Securin and Separase comprises a powerful prognostic tool to identify patients in risk of breast cancer death. In our scenario, the proposed model may facilitate personalized clinical decision suggesting less aggressive therapy for patients associated with low risk of mortality and indicating benefits from adjuvant therapy for a subgroup of patients with aggressive disease. Despite the accumulating data on multiparametric prognostic models, the clinical judgement remains the key determinant on selecting between the different treatment schemes.

\section{Supplementary information}

Supplementary information accompanies this paper at https://doi.org/10. 1186/s12885-020-07045-3.

Additional file 1. Details of $\mathrm{HC}$ for detecting Securin, Separase, Cdc20, Pttg1IP, SA2, Cdk1 and CyclinB1 and routine clinical biomarkers of breast cancer ER, PR, Ki-67 and HER2. The datasheet contains the details of the immunohistochemical methods used in this study.

\section{Abbreviations}

APC/C: Anaphase-Promoting Complex / Cyclosome; Cdc20: Cell division cycle 20; Cdk1: Cyclin-Dependent Kinase protein 1; Cl: Confidence interval; CyclinB1: G2/mitotic-specific cyclin-B1; ER: Estrogen receptor; ESPL1: Extra Spindle Pole Bodies Like protein 1; HER2: Human epidermal growth factor 2; HR: Hazard ratio; IHC: Immunohistochemistry; ISH: In situ hybridization; $\mathrm{N}+$ : Lymph node positive; N-: Lymph node negative; PBF: Pituitary tumortransforming gene 1 binding factor; PR: Progesterone receptor; Pttg1: Pituitary tumor-transforming gene 1; Pttg1IP: Pituitary tumortransforming gene 1 interacting protein; SA2: Stromal antigen 2; SAC: Spindle Assembly Checkpoint; TMA: Tissue microarray; TNBC: Triple negative breast cancer

\section{Acknowledgements}

The authors wish to thank Mrs. Sinikka Collanus for preparing the immunohistochemical stainings.

\section{Authors' contributions}

HR participated in the design, histological and immunohistochemical analyses and writing of the manuscript, EL performed the statistical analyses, SK and LK selected and provided the patient specimen from Auria biobank, TK selected and provided the patient specimen from the Biobank of Central Finland, KT participated in analyzing the data and writing the manuscript, PK supervised the study and prepration of the manuscript. All authors read and approved the final manuscript.

\section{Funding}

This study has been financially supported by the Cancer Society of Finland, Turku University Hospital and Finska Läkaresällskapet foundation.

The funding bodies played no role in the design of the study and collection, analysis, and interpretation of data and in writing the manuscript.

\section{Availability of data and materials}

The data that support the findings of this study are available from Auria Biobank (www.auriabiobank.fi), Turku University Hospital, Turku, and Biobank of Central Finland, Jyväskylä, Finland but restrictions apply to the availability of these data, which were used under license for the current study, and so are not publicly available. Data are however available from the authors upon reasonable request and with permission of the Biobanks stated above.

\section{Ethics approval and consent to participate}

The research was performed in accordance with the ethical standards of institutional and national research committees approved by the Regional Ethical Review Boards of Turku University Hospital and Auria Biobank, Turku, Finland, Central Hospital of Central Finland, Jyväskylä, Finland, and Finnish Cancer Registry, Cancer Society of Finland, Helsinki, Finland (permit numbers 6/2002, AB15-9859 and TK-53-716-16). All research procedures were performed in accordance with the 1964 Helsinki declaration and its amendments (www.wma.net/policies-post/wma-declaration-of-helsinki-ethicalprinciples-for-medical-research-involving-human-subjects) and the Finnish Biobank Act (688/2012, Ministry of Social Affairs and Health, Helsinki, Finland). The patients have been included in the study according to the Finnish Biobank Act 688/2012, Ministry of Social Affiars and Health (https://www.finlex.fi/ en/laki/kaannokset/2012/20120688). According to the Act, the national biobank organization, after ethical pre-evaluation, allows collection, storage and processing of patient specimen and information for medical research. 


\section{Consent for publication}

Not applicable.

\section{Competing interests}

The authors declare no competing interests.

\section{Author details}

${ }^{1}$ Institute of Biomedicine, University of Turku, Turku, Finland. ${ }^{2}$ Central Hospital of Central Finland, Jyväskylä, Finland. ${ }^{3}$ Department of Biostatistics, University of Turku, Turku, Finland. ${ }^{4}$ Turku University Hospital, Turku, Finland.

${ }^{5}$ Department of Pathology, University of Turku, Kiinamyllynkatu 10/MedD5A, 20500 Turku, Finland.

Received: 20 January 2020 Accepted: 5 June 2020

Published online: 16 June 2020

\section{References}

1. Wirapati P, Sotiriou C, Kunkel S, Farmer P, Pradervand S, Haibe-Kains B, et al. Meta-analysis of gene expression profiles in breast cancer: toward a unified understanding of breast cancer subtyping and prognosis signatures. Breast Cancer Res. 2008;10:R65.

2. Lee A, Djamgoz MBA. Triple negative breast cancer: emerging therapeutic modalities and novel combination therapies. Cancer Treat Rev. 2018;62:110-22.

3. Rakha EA, Soria D, Green AR, Lemetre C, Powe DG, Nolan CC, et al. Nottingham prognostic index plus (NPI+): a modern clinical decision making tool in breast cancer. Br J Cancer. 2014;110:1688-97.

4. Prat A, Pineda E, Adamo B, Galván P, Fernández A, Gaba L, et al. Clinical implications of the intrinsic molecular subtypes of breast cancer. Breast. 2015;24(Suppl 2):26-35.

5. Filipits $M$, Nielsen TO, Rudas M, Greil R, Stöger $H$, Jakesz $R$, et al. The PAM50 risk-of-recurrence score predicts risk for late distant recurrence after endocrine therapy in postmenopausal women with endocrine-responsive early breast cancer. Clin Cancer Res. 2014;20:1298-305.

6. Potapova T, Gorbsky GJ. The consequences of chromosome segregation errors in mitosis and meiosis. Biology. 2017;6:E12.

7. Musacchio A. The molecular biology of spindle assembly checkpoint signaling dynamics. Curr Biol. 2015:25:R1002-18.

8. Dominguez-Brauer C, Thu KL, Mason JM, Blaser H, Bray MR, Mak TW. Targeting mitosis in cancer: emerging strategies. Mol Cell. 2015;60:524-36.

9. Talvinen K, Tuikkala J, Nevalainen O, Rantanen A, Hirsimäki P, Sundström J, et al. Proliferation marker securin identifies favourable outcome in invasive ductal breast cancer. Br J Cancer. 2008;99:335-40.

10. Grizzi F, Di Biccari S, Fiamengo B, Štifter S, Colombo P. Pituitary tumortransforming gene 1 is expressed in primary ductal breast carcinoma, lymph node infiltration, and distant metastases. Dis Markers. 2013;35:267-72.

11. Solbach C, Roller M, Peters S, Nicoletti M, Kaufman M, Knecht R. Pituitary tumor-transforming gene (PTTG): a novel target for anti-tumor therapy. Anticancer Res. 2005:25:121-5.

12. Smith VE, Franklyn JA, McCabe CJ. Pituitary tumor-transforming gene and its binding factor in endocrine cancer. Expert Rev Mol Med. 2010;12:e38.

13. Karra H, Repo H, Ahonen I, Löyttyniemi E, Pitkänen R, Lintunen M, et al. Cdc20 and securin overexpression predict short-term breast cancer survival. Br J Cancer. 2014:110:2905-13.

14. Gurvits N, Löyttyniemi E, Nykänen M, Kuopio T, Kronqvist P, Talvinen K. Separase is a marker for prognosis and mitotic activity in breast cancer. $\mathrm{Br} J$ Cancer. 2017;117:1383-91.

15. Tang J, Lu M, Cui Q, Zhang D, Kong D, Liao X, et al. Overexpression of ASPM, CDC20, and TTK confer a poorer prognosis in breast cancer identified by gene co-expression network analysis. Front Oncol. 2019;9:e310.

16. Repo H, Löyttyniemi E, Nykänen M, Lintunen $M$, Karra H, Pitkänen R, et al. The expression of cohesin subunit SA2 predicts breast cancer survival. Appl Immunohistochem Mol Morphol. 2016;24:615-21.

17. Repo H, Gurvits $N$, Löyttyniemi E, Nykänen $M$, Lintunen $M$, Karra $H$, et al. PTTG1-interacting protein (PTTG1IP/PBF) predicts breast cancer survival. BMC Cancer. 2017;17:705-12.

18. Zhang N, Pati D. Biology and insights into the role of cohesin protease separase in human malignancies. Biol Rev Camb Philos Soc. 2017;92:2070-83.

19. Goldhirsch A, Ingle JN, Gelber RD, Coates AS, Thurlimann B, et al. Thresholds for therapies: highlights of the St Gallen international expert consensus on the primary therapy of early breast cancer 2009. Ann Oncol .2009;20:13191329.
20. Lakhani SR, Ellis IO, Schnitt SJ, Tan PH, van de Vijver MJ, editors. WHO classification of Tumours of the breast. 4th ed. Lyon: IARC; 2012. p. 10-1.

21. Coates AS, Winer EP, Goldhirsch A, Gelber RD, Gnant M, Piccart-Gebhart M, et al. Tailoring therapies - improving the management of early breast cancer: St Gallen international expert consensus on the primary therapy of early breast Cancer. Ann Oncol. 2015;26:1533-46.

22. A. Goldhirsch, EP, Winer AS, Coates AS, Gelber RD, Piccart-Gebhart M, Thürlimann $B$, et al. Personalizing the treatment of women with early breast cancer: highlights of the St Gallen International Expert Consensus on the Primary Therapy of Early Breast Cancer. Ann Oncol. 2013;24:2206-2223.

23. Suzuki T, Urano T, Miki Y, Moriya T, Akahira J, Ishida T, et al. Nuclear cyclin B1 in human breast carcinoma as a potent prognostic factor. Cancer Sci. 2007:98:644-51.

24. Wolff AC, Hammond MEH, Hicks DG, Dowsett M, McShane LM, Allison KH, et al. Recommendations for human epidermal growth factor receptor 2 testing in breast cancer: American Society of Clinical Oncology/College of American Pathologists clinical practice guideline update. Arch Pathol Lab Med. 2014;138:241-56.

25. Kamenz J, Hauf S. Time to split up: dynamics of chromosome separation. Trends Cell Biol. 2017;27:42-54.

26. Tong Y, Zhao W, Zhou C, Wawrowsky K, Melmed S. PTTG1 attenuates druginduced cellular senescence. PLoS One. 2011;6:e23754.

27. Meyer R, Fofanov V, Panigrahi A, Merchant F, Zhang N, Pati D. Overexpression and mislocalization of the chromosomal segregation protein separase in multiple human cancers. Clin Cancer Res. 2009;15:2703-10.

28. Shi R, Sun Q, Sun J, Wang X, Xia W, Dong G, et al. Cell division cycle 20 overexpression predicts poor prognosis for patients with lung adenocarcinoma. Tumour Biol. 2017;39:1-10.

29. Ding ZY, Wu HR, Zhang JM, Huang GR, Ji DD. Expression characteristics of CDC20 in gastric cancer and its correlation with poor prognosis. Int J Clin Exp Pathol. 2014;7:722-7.

30. Read ML, Fong JC, Modasia B, Fletcher A, Imruetaicharoenchoke W, Thompson RJ, et al. Elevated PTTG and PBF predicts poor patient outcome and modulates DNA damage response genes in thyroid cancer. Oncogene. 2017;36:5296-308.

31. Yang W, Cho H, Shin HY, Chung JY, Kang ES, Lee EJ, et al. Accumulation of cytoplasmic Cdk1 is associated with cancer growth and survival rate in epithelial ovarian cancer. Oncotarget. 2016;7:49481-97.

32. Sung WW, Lin YM, Wu PR, Yen HH, Lai HW, Su TC, et al. High nuclear/ cytoplasmic ratio of Cdk1 expression predicts poor prognosis in colorectal cancer patients. BMC Cancer. 2014;14:951-7.

33. Sun X, Zhangyuan G, Shi L, Wang Y, Sun B, Ding Q. Prognostic and clinicopathological significance of cyclin B expression in patients with breast cancer: a meta-analysis. Medicine. 2017;96:e6860.

34. Yerushalmi R, Woods R, Ravdin PM, Hayes MM, Gelmon KA. Ki67 in breast cancer: prognostic and predictive potential. Lancet Oncol. 2010;11:174-83.

35. Penault-Llorca F, Radosevic-Robin N. Ki67 assessment in breast cancer: an update. Pathology. 2017:49:166-71.

36. Phung MT, Tin Tin S, Elwood JM. Prognostic models for breast cancer: a systematic review. BMC Cancer. 2019;19:230-47.

37. Green AR, Soria D, Stephen J, Powers DG, Nolan CC, Kunkler I, et al. Nottingham prognostic index plus: validation of a clinical decision making tool in breast cancer in an independent series. J Pathol Clin Res. 2016;2:3240.

38. Albergaria A, Ricardo S, Milanezi F, Carneiro V, Amendoeira I, Vieira D, et al. Nottingham prognostic index in triple-negative breast cancer: a reliable prognostic tool? BMC Cancer. 2011;11:299-306.

\section{Publisher's Note}

Springer Nature remains neutral with regard to jurisdictional claims in published maps and institutional affiliations. 